Virginia Commonwealth University VCU Scholars Compass

1990

\title{
Effect of size and dimensionality on the magnetic moment of transition metals
}

Feng Liu

Virginia Commonwealth University

S. N. Khanna

Virginia Commonwealth University

P. Jena

Virginia Commonwealth University

Follow this and additional works at: http://scholarscompass.vcu.edu/phys_pubs

Part of the Physics Commons

Liu, F., Khanna, S. N., Jena, P. Effect of size and dimensionality on the magnetic moment of transition metals. Journal of Applied Physics 67, 4484 (1990). Copyright (C 1990 AIP Publishing LLC.

\section{Downloaded from}

http://scholarscompass.vcu.edu/phys_pubs/135

This Article is brought to you for free and open access by the Dept. of Physics at VCU Scholars Compass. It has been accepted for inclusion in Physics Publications by an authorized administrator of VCU Scholars Compass. For more information, please contact libcompass@vcu.edu. 


\title{
Effect of size and dimensionality on the magnetic moment of transition metals
}

\author{
Feng Liu, S. N. Khanna, and P. Jena \\ Physics Department, Virginia Commonwealth University, Richmond, Virginia 23284-2000
}

\begin{abstract}
The effect of size and dimensionality on the magnetic moments of $\mathrm{Fe}, \mathrm{Co}$, and $\mathrm{Ni}$ have been studied theoretically by confining the atoms to various structural forms such as chains, surfaces, and thin fims. The size of these systems is controlled by limiting the number of atoms. A new frst-principles theory is developed that enables us to study the electron spin density of states and moments of atoms in clusters containing two to a few thousand atoms. The theory is based upon the elementary principles governing the tight binding and linear combination of atomic orbitals formulations. It contains no adjustable parameters and can be applied to systems with or without topological symmetry. We have discovered quantum size effects on the magnetic moments of linear chains and these effects disappear when the chains contain more than 20 atoms. We have also found distinct effects of the local environment on the magnetic moment. For example, the moments increase with decreasing coordination number and increasing interatomic distance. Our results will be compared with available experimental and theoretical results.
\end{abstract}

\section{MTROOUCTION}

Study of magnetism is one of the ancient fields of research. In spite of numerous experiments and theories dedicated to understanding the origin of magnetic order, the interest in this field still remains strong. Part of this arises from the fact that magnetic materials play an important role in our technology and consequently there is a constant search for finding ways to produce new magnetic materials. These studies have been possible through developments and new techniques of preparation such as molecular-beam epitaxy ${ }^{1}$ (MBE), cluster beams, ${ }^{2}$ and techniques of characterization such as scanning tunneling microscopy. ${ }^{3}$ One can produce materials in layers of varying thickness and clusters of varying sizes. The magnetic properties of these materials depend on their dimensionality (chains, planes, and fims) and size (microciusters, nanocrystais).

Experiments in the past few years have concentrated on these novel magnetic materials because of the continuing demand by modern technology and by the limitations posed by nature on the number of elemental ferromagnets available. While these experimental studies have lead to a new era in the research on magnetism, an understanding of how and why the environment of the material has a controling influence on the magnetic moment remains primarily an unsolved puzzle. In the past few decades, impressive developments $^{5-7}$ have been made in designing theories that can predict magnetic moments of clean surfaces and bulk with quantitative accuracy. These theories, however, rely on the symmetry of the system and make use of the Bloch theorem. Unfortunately, such methods have limited use when one has to deal with a particle of arbitrary size and composition. One needs to (a) develop a theory that is versatile enough to be used in systems with lower symmetry and dimension and (b) to be assured that such a theory can predict magnetic moments with quantitative accuracy. In this paper we formulate a theory from first principles and show how the basic interactions in a dimer can be used to calculate the spin den- sity of states, and hence the magnetic moment of a wide range of systems starting from clusters to crystals. The accuracy of its prediction can be tested against those from well established state-of-the-art $a b$ initio theoretical techniques. We then proceed to study the magnetic moments of hitherto unexplored novel systems. We find correlations between the size, dimensionality, and topology of a system and its magnetic moment. In Sec. 11, we outlined briefly our theoretical method and discuss results on a few chosen systems in Sec. III. Section IV contains a brief summary of conclusions.

\section{THEORETICAL FORMULATION}

Our method ${ }^{8}$ is based upon a cross between the tightbinding theory ${ }^{9}$ and the linear combination of atomic orbitals (LCAO). ${ }^{10}$ The former determines the density of states in terms of tight-binding parameters. Those are obtained from first-principles using the latter method applied to a dimer. Thus, the combined tight-binding LCAO technique, referred to as the $a b$ initio tight-binding theory (ATB), contains no adjustable parameters and needs no experimental input to calculate the electronic structure. The method is based upon a real-space technique and, therefore, is not limited to systems of high symmetry and long-range order.

We start with the expression for the density of states, $n_{j}^{\lambda}(E)$, with $j$ as the site and $\lambda$ as the orbital index. The density of states for each spin can be obtained by averaging over the partial density of states, $n_{j}^{\lambda}(E)$, namely

$$
n_{j}(E)=\frac{1}{M} \sum_{i=1}^{M} n_{j}^{\lambda}(E),
$$

where $M$ is the number of orbitals. The partial density of states can be expressed using the moment and the continued fraction approach, ${ }^{11}$

$$
n_{j}^{\lambda}(E)=-\frac{1}{\pi} \operatorname{Im} G_{j}^{\lambda}(E+i \epsilon) .
$$


The Green's function $G(E)$ is related to the monents $\mu_{n}$ of the density of states by

$$
\begin{aligned}
G_{j}(E) & =\sum_{p=0}^{\infty} \frac{\mu_{p}^{j, \lambda}}{E^{p+1}} \\
& =\frac{1}{E-a_{1}^{j}-\frac{b_{1}^{j}}{E-a_{2}^{j}-\frac{b_{2}^{j}}{\theta_{0}}}} .
\end{aligned}
$$

Here $a^{j}$ and $b^{j}$ are continued fraction coeficients related to the moments $\mu_{n}^{j}$. The moments $\mu_{p}^{i, \lambda}$ are given by the relation

$$
\begin{aligned}
\mu_{p}^{i, \lambda}= & \frac{1}{N} \sum_{\substack{j, k, \ldots \\
\mu, \gamma, \ldots}}\langle i, \lambda|H| j, \mu\rangle\langle j, k|H| k, v\rangle \\
& \times\langle k, v|H \cdots H| i, \lambda\rangle .
\end{aligned}
$$

In conventional applications of the tight-binding technique ${ }^{9}$ the off-diagonal matrix elements in Eq. (5) between adjacent sites are the only ones that are taken into account and are obtained by fitting known band-structure results. The problem with such a procedure is twofold. First, the parameters depend upon the data they were fitted to and secondly, are not easily transferable to other systems. ${ }^{12}$ However, this technique has the merit of being computationally very easy.

We have developed a technique where the parameters involving the matrix elements $\langle i, \lambda|H| j, \mu\rangle$ can be obtained from first principles. We show ${ }^{8}$ that if one were to evaluate these matrix elements by solving a self-consistent Schrödinger equation for a dimer in the LCAO framework and use them in the tight-binding equation (5), the resuiting density of states agrees well with the conventional band-structure results. Furthermore, these parameters are transferable to other systems with differing topology and symmetry. The only requirement is that these parameters are to be obtained for interatomic distances appropriate to the system under consideration. In the LCAO framework, the molecular spin orbital is given by

$$
\psi=\sum_{j \alpha} \mathrm{C}_{j \alpha}\lfloor; \alpha\rangle
$$

where $\langle j, \alpha\rangle$ are the atomic orbicals of site $j$ and orbital index $\alpha$. The variational coefficients $C_{j \alpha}$ are obtained by solving the Rayleigh-Ritz equation

$$
(\mathrm{H}-E \mathrm{~S}) \mathrm{C}=0
$$

where $H$ and $S$ are the Hamiltonian and overlap matrices and $E$ is the eigenvalue. With $C_{j c}$ determined self-consistently, the matrix elements in Eq. (5) can be evaluated in a straightforward manner. The magnetic moment can now be obtained by integrating the spin density of states.

\section{RESULTS AND DISCUSSIONS}

The Slater-Koster parameters have been obtained for $\mathrm{Fe}, \mathrm{Co}$, and $\mathrm{Ni}$ using the density functional theory and the discrete variational method? (DVM) for dimers as a function of interatomic distances. These were fitted to a form

$$
X=X_{0} \exp \left[-Q\left(R-R_{0}\right) / R_{0}\right],
$$

which gives the dependence of the parameters on interato- mic distance $R . R_{0}$ defines the nearest-neighbor distance of the atoms in the bulk and $X_{0}$ is the corresponding tightbinding parameter. The exponent $Q$ and $X_{0}$ and $R_{0}$ have been tabulated by us in an earlier publication. ${ }^{8}$ We have verified that the density of states obtained using the formulation described here predicts the saliant features in bulk band structure data. The magnetic moments calculated by our method for $\mathrm{Fe}, \mathrm{Co}$, and $\mathrm{Ni}$ are, respectively, $2.53 \mu_{B}, 1.69 \mu_{B}$, and $0.59 \mu_{B}$. These agree very well with the corresponding experimental values of $2.2 \mu_{B}, 1.6 \mu_{E}$, and $0.59 \mu_{E}$. The moments on various layers of $\mathrm{Fe}(100)$ slabs and $\mathrm{Ni}(100)$ and Ni (111) slabs also agree very well with full potential linearized augmented plane-wave (APW) (FLAPW) results. ${ }^{5}$ In Fig. 1 we present our results of the magnetic moments $\Delta \mu$ defined with respect to the bulk moment $\mu_{0}$, namely, $\Delta \mu=\mu-\mu_{0}$ for $\mathrm{Fe}, \mathrm{Co}$, and $\mathrm{Ni}$ confined to various topolog. ical forms. These include linear chains, surfaces, thin films of different crystallographic directions, and bulk. The number of nearest-neighbor atoms in these systems vary over a wide range. For example, in $F e$, the number of nearest neighbors in a linear chain, (100) monolayer, and bulk, are, respective$1 \mathrm{y}, 2,4$, and 14 . These rumbers are commonly referred to as coordinate number ( $\mathrm{CN}$ ), and can be varied by simulating different structural arrangements. For example, a monovacancy in $\mathrm{Fe}$ will reduce the $\mathrm{CN}$ from 14 to 13 . In $\mathrm{Ni}$, the coordination numbers of $2,4,6,8,9$, and 12 can be obtained from linear chains, (100) monolayer, (111) monolayer, (100) surface, (111) surface and bulk respectively. Note that the moments $\Delta, l$ decrease systematically as the coordination numbers increase. This decrease in the moment is caused by broadening of the density of states as the orbitals of increasing nearest neighbors overlap with the probe atom. Consequently, the atom is most magnetic and the bulk is least magnetic. The broadening of the censity of states (DOS) can also be caused by changing the interatomic separation. Reducing the distance between two magnetic atoms will cause the overlap to increase and the moments to decrease. It is possible to artificially separate two magnetic atoms to a distance longer than they would like to be in the bulk by putting them on substrates of appropriate latice

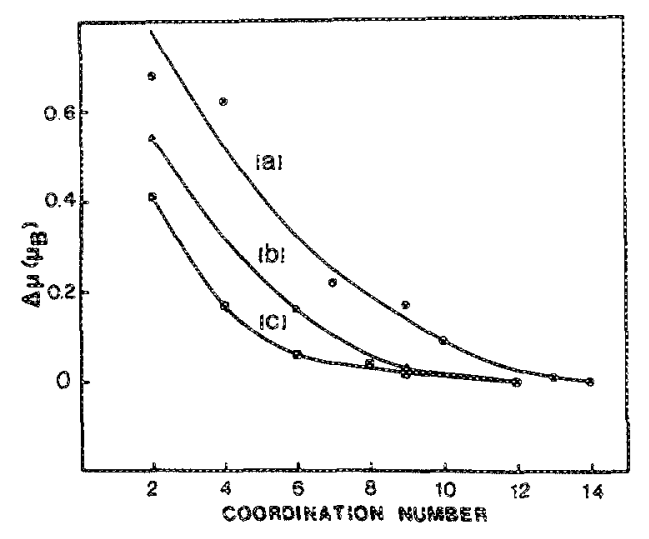

FIG. 1. Deviation from the bulk magnetic moment $\Delta \mu$ in Fe, Co, and Ni as a function of nearest coordination number (in various structures). (a), (b), and (c) correspond to $\mathrm{Fe}, \mathrm{Co}$, and $\mathrm{Ni}$, respectively. The smooth lines are drawn simpiy to guide the eye. 
spacing. Such techniques called inverse pressure is currently being used with the aid of MBE apparatus.

We now discliss the magnetic moments of $\mathrm{Fe}, \mathrm{Co}$, and $\mathrm{Ni}$ atoms confined to linear chains of varying length. The coordination number of an atom in a chain is two, and if the nearest neighbor is the only important factor on the moment, one would think that the moment of the probe atom would not depend on the chain length. Our results of the moment per atom as a function of the total number of atoms in the chain (e.g., chain length) in Fig. 2 speak to the contrary. The moments oscillate with size and converge to the infinite limit rather quickly. The results in Ni are most revealing. Note that here the moment per atom in chains containing an odd number of atoms is larger than the adjacent chains having an even number of atoms. The origin of this odd-even alternation in the moments can be understood by calculating the spin density of states. We found that for even atom chains the bonding and antibonding states are well separated from the $d$ manifold. For odd atom chains, on the other hand, a nonbonding $s$-state occurs in the center of the $d$ manifold below the Fermi energy. This nonbonding $s$ band has to be filled by transferring electrons from the minority $d$ band. Consequently, the moment is enhanced over that in the even atom chain. It is this $s-d$ mixing that is responsible for the moment fluctuation at small sizes. As the size of the chain increases, the broadening of the levels reduces the difference in $s-d$ mixing between odd and even atom chains. Thus, one winnesses the disappearance of the quantum size effect.

The results in Fig. 2 can also be used to answer another important question concerning the adequacy of modeling infinite systems with finite clusters. One often wonders if the size of a chuster has been large enough so that the boundary does not affect the calculated properties. It is difficult to find an unambiguous answer using first principles theory due to the heavy demand on computer time. However, using our ATB method, we can easily calculate the electronic structure of a few thousand atoms. Our results in Fig. 2 indicate that the moments of chains converge with as few as 20 atoms.

We should stress that the moments in Fig. 2 are based upon a non-self-consistert theory, although it contains no adjustable parameters. Since there are no experiments on chains with which our prediction can be compared, it is legitimate to question the quantitative accuracy of our prediction in Fig. 2. We have, therefore, repeated the calculations of moments for Ni chains containing 2,3,4,5,6, and 7 atoms using the self-consistent LCAO-MO (molecular orbital) theory within the discrete variation method (DVM) scheme. ${ }^{7}$ Our results using the ATB theory for the moments of $2,3,4,5,6$, and 7 atom chains are, respectively, $1.0 \mu_{H *}$, $1.32 \mu_{B}, 1.0 \mu_{B}, 1.19 \mu_{B}, 1.0 \mu_{B}$, and $1.12 \mu_{B}$. These are in excellent agreement with the corresponding results based on the self-consistent DVM, namely, $1.0 \mu_{E F}, 1.31 \mu_{B}, 1.0 \mu_{B}$, $1.17 \mu_{B}, 1.0 \mu_{B}$, and $1.14 \mu_{B}$.

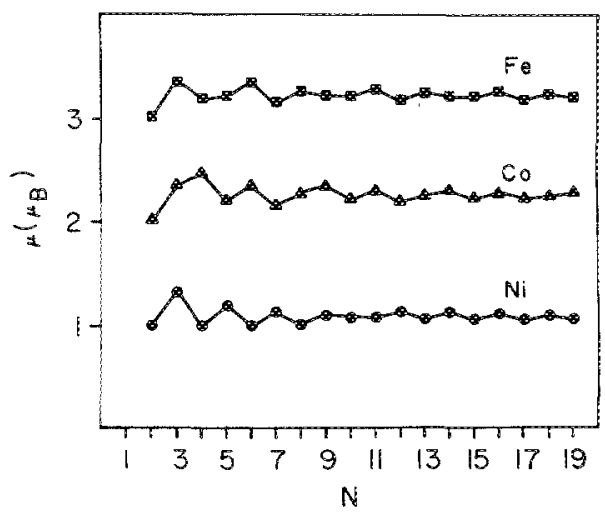

FIG. 2. Magnetic moment $\mu$ in $\mathrm{Fe}, \mathrm{Co}$, and $\mathrm{Ni}$ chains as a function of number of atoms in the chain.

In conclusion, we have formulated a new scheme to calculate the electronic structure and magnetic properties of a wide range of systems with quantitative accuracy and little demand on computer time. Using this method we have discovered the quantum size effect on the magnetism of finite systems and the role of coordination number of magnetic moments.

\section{ACKNOWLEDGMENT}

This work was supported in part by a grant from the Army Research Office (DAAL 03-89-K-0015).

'E. Kaldis, Ed., Crystal Growth of Electronic Materials (North-Holland, Ansterdam, 1985).

${ }^{2}$ P. Jena, B. K. Rao, and S. N. Khanna, Physics and Chemisiry of Small Clusters (Plenum Press, New York, 1987).

${ }^{3}$ G. Binning and H. Rohrer, Rev. Mod. Phys. 59, 615 (1987).

${ }^{4}$ D. M. Cox, D. J. Trevor, R. L. Whetten, E. A. Rohifing, and A. Kaldor, Phys. Rev. B 32, 7290 (1985); M. Stampanoni, A. Vaterlaus, D. Pescia, M. Aeschlimann, and F. Meier, ibid. 37, 10380 (1988).

${ }^{5}$ S. Ohnishi, A. J. Freeman, and M. Weinert, Phys. Rev. B 28, 6741 (1983); H. Krakauer, A. J. Frseman, and E. Wimmer, ibid. 28, 610 (1983); A. J. Freeman, J. H. Xu, and T. Jarlborg, J. Magn. Magn. Mater. 31, 909 (1983); A. J. Freeman, ibid. 35, 31 (1984).

${ }^{6} O$ Jepson, J. Madsen, and O. K. Anderson, Phys. Rev. B 26, 2790 (1982).

${ }^{7}$ D. E. Ellis, Int. J. Quantum Chem. S2, 35 (1968); D. E. Ellis and G. S. Painter, Phys. Rev. B 2, 2887 (1970).

${ }^{8}$ F. Liu, M. R. Press, S N. Khanna, and P. Jena, Phys. Rev. B 39, 6914 (1989).

${ }^{9}$ D. A. Papaconstantopoulos, Handbook of the Eand Structure of Elemental Solids (Plenum, New York, 1986).

${ }^{10 J}$. C. Slater, The Seif-Consistent Field for Molecules and Solids (McGrawHill, New York, 1974), Voi. 4.

${ }^{11}$ F. Cryot-Lackmann and S. N. Khanna, in Excitations in Disordered Systems, edited by M. F. Thorp (Plenum, New York, 1980); H. Ehrenreich, F. Seitz, and D. Turnbuli, Eds. Solid State Physics (Academic, New York, 1980), Vol. 35.

12 A. Weinert and A. J. Freeman, J. Magn. Magn. Mater, 38, 23 (1983); C. L. Fu and A. J. Freenan, ibid. 34, 777 (1986). 